\title{
Identification of essential genes for Escherichia coli aryl polyene biosynthesis and function in biofilm formation
}

Isabel Johnston ${ }^{1}$, Lucas J Osborn ${ }^{1,2 *}$, Rachel L Markley ${ }^{1 *}$, Elizabeth A McManus ${ }^{1,3}$, Anagha 5 Kadam $^{1}$, Karlee B Schultz ${ }^{1,4}$, Nagashreyaa Nagajothi ${ }^{1,5}$, Philip P Ahern ${ }^{1,2,6}$, J Mark Brown ${ }^{1,2,6}$, Jan Claesen ${ }^{1,2,6+}$

${ }^{1}$ Department of Cardiovascular and Metabolic Sciences, Lerner Research Institute, Cleveland Clinic, Cleveland, $\mathrm{OH}$, USA

10 '2Department of Molecular Medicine, Cleveland Clinic Lerner College of Medicine of Case Western Reserve University, Cleveland, $\mathrm{OH}$, USA

${ }^{3}$ Current affiliation: National Cancer Institute, National Institutes of Health, Bethesda, MD, USA

${ }^{4}$ College of Arts and Sciences, John Carroll University, University Heights, OH, USA

5University Honors College, University of Pittsburgh, Pittsburgh, PA, USA

$15{ }^{6}$ Center for Microbiome and Human Health, Cleveland Clinic, Cleveland, OH, USA

*LJO and RLM contributed equally to this work

†Correspondence to: claesej@ccf.org 


\section{ABSTRACT}

Aryl polyenes (APEs) are specialized polyunsaturated carboxylic acids that were identified in silico as the product of the most widespread family of bacterial biosynthetic gene clusters (BGCs). They are present in several Gram-negative host-associated bacteria, including multi-drug resistant human pathogens. Here, we characterize a biological function of APEs, focusing on the BGC from a uropathogenic Escherichia coli (UPEC) strain. We first perform a genetic deletion analysis to identify the essential genes required for APE biosynthesis. Next, we show that APEs function as fitness factors that increase protection from oxidative stress and contribute to biofilm formation. Together, our study highlights key steps in the APE biosynthesis pathway that can be explored as potential drug targets for complementary strategies to reduce fitness and prevent biofilm formation of multi-drug resistant pathogens.

\section{INTRODUCTION}

In a global search for novel types of bacterial secondary metabolites, we previously identified aryl polyenes (APEs) as the products of the most abundant biosynthetic gene cluster (BGC) family [1]. APEs are present in most major bacterial genera throughout the Proteobacteria and Bacteroidetes. They are often found in host-associated bacteria, including commensals as well as pathogens of humans, animals, and plants [1]. Among these are several human pathogens that are a major concern in nosocomial multidrug-resistant infections, including Acinetobacter baumannii, Burkholderia cenocepacia, as well as several Enterobacter isolates [2, 3]. APE BGCs are also present in most types of pathogenic E. coli, whereas they are typically absent from commensal and laboratory strains of E. coli [1].

APEs produced by diverse bacterial genera share a remarkably similar chemical scaffold, consisting of an aryl head group conjugated to a polyene carboxylic acid tail [1, 4-7] (Fig. 1). The main differences between APEs of different bacterial genera are in the polyene chain length and in the hydroxylation, methylation, or halogenation of the head group. This structural similarity is reflected in the observation that phylogenetically diverse APE BGCs share conserved core gene functions, resembling biosynthesis machinery for fatty acids and type II polyketides. Notably, an in silico comparison between BGCs revealed differential enzymatic functions in genus-dependent mechanisms for biosynthesis initiation, cell envelope attachment, and head group tailoring. APE head groups can be tailored by methylation (as in the E. coli CFT073 APE $\mathrm{Ec}_{\mathrm{c}}$ and flexirubin [1, 8, 9]) and halogenation (as in xanthomonadin [10]), while the hydroxyl group is commonly derived from the corresponding precursor molecule. While the core APE metabolites are similar in structure, further investigations are required to determine whether APEs also share a conserved lipid anchor in the outer membrane. Heterologous expression of the BGCs from E. coli CFT073 $\left(\mathrm{APE}_{\mathrm{Ec}}\right)$ and Vibrio fischeri ES114 (APE $\mathrm{Vf}_{\mathrm{f}}$ confirmed that these BGCs encompass all genes required for APE biosynthesis [1]. Moreover, the biosynthesis of the core APE moiety from the nematode symbiont Xenorhabdus doucetiae DSM17909 (APExd) was recently reconstituted in vitro by Grammbitter et al. [11]. The functions of nine enzymes from the APE $\mathrm{Xd}_{\mathrm{d}}$ BGC were

40 characterized after heterologous expression and purification from $E$. coli and used to form APEXd in vitro. Our current study addresses outstanding questions in APE biology, including the identity of the essential genes for in vivo biosynthesis and uncovering their biological function among the bacteria that produce them.

APEs are covalently attached in the Gram-negative outer membrane [12], allowing the bacteria that produce them to potentially alter interactions with the surrounding environment. Among the best studied bacterial lifestyle changes that involve alterations in the expression of cell envelope structures are aggregation, adhesion to surfaces, and biofilm formation. Biofilms form when 
bacteria switch from planktonic growth to an adherent, three-dimensional lifestyle by embedding themselves in a self-produced extracellular matrix [13]. Biofilm formation impacts bacterial physiology, affecting pathogenesis and antibiotic resistance. Decreased antibiotic susceptibility can be due to a slowing of bacterial growth and metabolic activity, as well as reduced ability of antibiotic molecules to penetrate the biofilm matrix and reach interior cells [14, 15]. Moreover, growth in biofilms is associated with horizontal gene transfer, leading to more rapid strain adaptation and evolution. Since pathogens are more resilient when growing in biofilms, understanding the underlying mechanisms of biofilm formation and devising strategies to prevent and eliminate this process represent an important approach to limiting their pathogenic effects.

Gram-positive bacterial pathogens attach different types of polyenes in their cell envelope, including staphyloxanthin and granadaene (Fig. 1), which are polyenic virulence factors localized in the cell envelope of Staphylococcus aureus and Group B Streptococcus, respectively [16, 17]. Staphyloxanthin is a 'golden' carotenoid pigment produced from the mevalonate pathway, and granadaene is a red ornithine rhamno-polyene produced by fatty acid-like biosynthetic machinery $[16,17]$. Despite their different biosynthetic origins, both of these compounds share remarkable analogous functions: they protect their producers from damage induced by reactive oxygen species (ROS) [18, 19]. Exogenous ROS can originate from a variety of sources, such as UV light, certain antibiotics or the host immune system. Neutrophils and macrophages utilize ROS and reactive nitrogen species (RNS) production as an initial line of defense against invading bacterial pathogens [20]. The lethal effect of ROS can be attributed to widespread damage to bacterial cells via induction of DNA double strand breaks and oxidation of proteins and lipids [21, 22]. Polyenes that are localized in the cell envelope can scavenge external ROS, thereby preventing more extensive damage to essential cellular molecules and increasing bacterial fitness. Hence, characterization of the staphyloxanthin biosynthetic pathway has fueled the development of an anti-virulence strategy for managing $S$. aureus infections [23].

In this study, we focus on the functional characterization of the APE $\mathrm{Ec}_{\mathrm{C}} \mathrm{BGC}$ from uropathogenic $E$. coli. Using a heterologous expression and gene deletion approach, we identify the genes in the $B G C$ that are required for $A P E_{E c}$ biosynthesis. Bacterial strains that express $A P E_{E c}$ are conferred with a fitness advantage compared to their APE counterparts. APE $E_{E c}$ producers have an increased viability when subjected to an acute oxidative stress challenge and have an increased capability to form biofilms. Our work marks an important step in the biological understanding of $A P E_{E c}$ function and paves the way for the development of complementary strategies to reduce in vivo fitness and biofilm formation of multidrug-resistant human pathogens.

\section{RESULTS}

\section{The $E$. coli $\mathrm{APE}_{\mathrm{Ec}} \mathrm{BGC}$ contains predicted redundant functions and a dedicated transport system}

We previously identified APEs as the product of one of the most abundant bacterial BGC families and chose the BGC from E. coli CFT073 for in-depth characterization. Heterologous expression of this 18 gene, $15.5 \mathrm{~kb} B G C$ showed that this region contains all the genes required for production of $A P E_{E c}$ (Fig. 2A, [1]). All genes in the cluster are in the same orientation and several have overlapping open reading frames, suggesting the cluster is transcribed as one or multiple polycistronic mRNAs. The $A P E_{E C} B G C$ encodes some seemingly duplicated functions, for example there are two acyl carrier proteins (ACPs) (ApeE and F), two 3-hydroxyacyl-ACP dehydratases (Apel and P) and three 3-ketoacyl-ACP synthases (ApeC, O and R, Fig. 2, Table 1). In vitro studies by Grammbitter et al. [11] on the APExd $B G C$ showed that ApeR is required for initiation and that ApeC functions as a chain length factor through heterocomplex formation with 
ApeO, which is responsible for chain elongation. Interestingly, the ApeC chain length factor is encoded near the beginning of the cluster, whereas most of the other core biosynthesis genes are located quite distantly, near the cluster's end.

The $A P E_{E c}$ biosynthetic pathway is predicted to start with the activation of 4-hydroxybenzoic acid (4-HBA) by the specialized acyl-ACP synthetase ApeH (Fig. 3). In vitro reconstitution of the ApeH activity from the APE $E_{x d} B G C$ revealed that this enzyme is capable of 4-HBA adenylation and ACPloading without the formation of an intermediate CoA-thioester [11]. In E. coli and other Gramnegative bacteria, 4-HBA is produced by chorismate lyase (UbiC) as the first step in the ubiquinone biosynthesis pathway [24]. The $\mathrm{APE}_{\mathrm{Ec}}$ cluster does not contain a dedicated phosphopantetheinyl transferase (PPTase) to activate the ACPs, ApeEF. An orphan PPTase, AcpT, was previously identified in E. coli O157:H7 EDL933, and in vitro biochemical analysis showed it can activate the respective ApeEF homologues in that strain [25]. The aromatic starter is further elongated with malonyl-ACP units by repetitive action of core biosynthetic enzymes related to those involved in fatty-acid and type II polyketide biosynthesis (Fig. 3). Methylation of the $A P E_{E c}$ aromatic head group is likely catalyzed by the S-adenosyl-L-methionine dependent methyltransferase ApeB. The exact timing of this methylation step has not yet been determined and as a result of in vitro analysis of the APExd methyltransferase, Grammbitter et al. postulated that methylation occurs post-APE biosynthesis [11]. The putative thioesterase ApeK could be

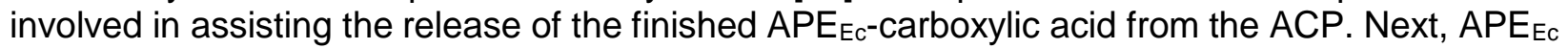
is predicted to be acylated to a larger molecular entity in the membrane by two acyltransferases (predicted glycerol-3-phosphate acyltransferase ApeD and glycosyltransferase ApeJ), possibly via a glycerophospholipid-derived intermediate. Finally, the APE-containing molecule is localized to the outer membrane via a dedicated transport system comprised of ApeM, a "resistancenodulation-division" (RND) family transmembrane protein and 'localization of lipoprotein' (Lol) homologues ApeL and ApeN (Table 1, [12, 26, 27]).

\section{Genetic analysis of the $A P E_{E c}$ BGC reveals essential biosynthesis genes}

We have constructed deletion mutants to link the individual genes in the APE $E_{E C} B G C$ to their role in the biosynthetic pathway and identify potential functionally redundant genes in vivo (Table 1). To avoid potential polar effects of gene deletions within polycistronic operons, we used an inframe "scarred" strategy involving a two-step PCR-generated recombineering procedure (Fig. S1, [28]). In the first step, the coding sequence between the start and stop codon for a gene of interest is replaced using a PCR cassette containing an antibiotic resistance marker with flanking FRT sites and homologous regions to the target gene. For overlapping genes, we designed in-frame deletion constructs that keep the entire overlapping open reading frame intact. In the second step, Flp recombinase is used to excise the resistance marker by recombining the FRT sites. In the resulting deletion mutant, the gene's coding region is replaced by an in-frame 81 nucleotide "scar" sequence that lacks stop codons in all reading frames, thereby avoiding potential polar effects on downstream genes.

Upon heterologous introduction of the $15.5 \mathrm{~kb} A P E_{\mathrm{Ec}} \mathrm{BGC}$ in a standard $E$. coli Top10 cloning strain (devoid of native BGC), we observed a strong yellow pigmentation phenotype [1]. Since APEs are covalently attached to the outer membrane [12], pigmentation is cell-associated and does not diffuse out into the culture medium. We used cell pellet pigmentation as a read-out for $A P E_{E c}$ pathway functionality: mutant plasmids that conferred wild-type level pigmentation, comparable to the pJC121 containing parent strain are designated $\mathrm{APE}^{+}$; mutant plasmids that conferred reduced pigmentation are $\mathrm{APE}^{ \pm}$; and mutant plasmids that did not display any pigmentation and thus were comparable to E. coli Top10 harboring the SuperCosl empty vector 
control were designated APE- (Fig. 2A, B). As expected, most mutants in predicted core biosynthetic genes (represented in shades of blue in Fig. 2A) are APE- Only deletion of apeA, the first gene in the BGC, retains wild type levels of pigmentation, suggesting it is not required for $A P E_{E c}$ biosynthesis. Surprisingly, deletion of one of the two ACPs, $\triangle a p e F$, retains partial pigmentation. Both acyltransferase mutants, $\triangle a p e D$ and $\triangle a p e J$, retained partial pigmentation as well. The latter observation suggests the formation of intermediates in the cell envelope attachment process. We did not obtain scarred deletion mutants for apeL, apeN or apeO and the APE- phenotype of their respective $\triangle a^{2} r^{R}$ mutants could be due to polar effects on essential downstream genes in the BGC. In summary, individual deletions in most of the core biosynthesis and transport genes abolished APE-associated pigmentation (Table 1 and Fig. 2), whereas deletion of genes that are predicted to occur later in the pathway result in reduced pigmentation that could be caused by reduced flux through the pathway and improperly attached APE $E_{\mathrm{Ec}}$ intermediates.

\section{Mutants that retain pigmentation produce biosynthetic or attachment intermediates}

To characterize potential $A P E_{E c}$ intermediates produced by our mutant strains, we performed a crude extraction of their cell pellets (Fig. S2) and profiled the APE-related metabolites in these extracts by high-performance liquid chromatography (HPLC; Figs. 4, S3). The primary APE $\mathrm{Ec}_{\mathrm{c}}$-lipid eluting near $100 \% \mathrm{ACN}$ only seems to occur in the $\mathrm{APE}^{+}$and $\triangle$ ape $R$ extracts. The $\triangle a p e A, \Delta a p e B$ and $\triangle a p e K$ strains produce a comparable set of immature APE carboxylic acids that could be early intermediates formed before commencing the attachment pathway (Fig. 4). This was a remarkable observation provided the near wild-type levels of pigmentation in the $\triangle a p e A$ strain. Our HPLC data indicate that ape $A$ is not obsolete for full assembly of the APE $E_{\text {cclipid. }} \triangle a p e D$, $\triangle a p e J$ and $\Delta a p e R$ produce late pathway intermediates, which likely consist of APE-carboxylic acids that are impaired in their proper attachment to the final membrane lipid anchor. Deletion of the apeF ACP (orange) results in the formation of a series of yellow pigmented intermediates, thus indicating it is not strictly required for biosynthesis of the APE $E_{E c}$-carboxylic acid moiety. We did not detect any APE-related metabolites in the remaining 9 of the 18 mutant strains (Fig. S3). This was surprising for $\triangle a p e M$ (transporter) since that strain's extract retained a low level of yellow pigmentation (Fig. S2). In summary, except for ape $R$, all genes in the BGC seem required for full assembly of the APE $\mathrm{Ec}_{\mathrm{C}}$-carboxylic acid or proper attachment to its membrane anchor.

\section{$A P E_{E c}$ confers increased protection from oxidative, but not nitrosative challenge}

Inspired by studies showing that staphyloxanthin deletion mutants of $S$. aureus are more susceptible to ROS [18, 29], we hypothesized that APEs fulfill a similar role for their Gramnegative producers. To test for protection against acute ROS stress, we compared $\mathrm{APE}^{+}$and APE- $E$. coli resilience in a hydrogen peroxide $\left(\mathrm{H}_{2} \mathrm{O}_{2}\right)$ survival assay (Fig. 5A). Bacteria from an overnight liquid culture were challenged with incremental concentrations of $\mathrm{H}_{2} \mathrm{O}_{2}$ for one hour, after which the reaction was quenched by addition of excess catalase. Dilutions of the reaction

40 mixtures were plated to count colony forming units and percent survival was calculated relative to the phosphate buffered saline (PBS) control incubated without added $\mathrm{H}_{2} \mathrm{O}_{2}$. At higher $\mathrm{H}_{2} \mathrm{O}_{2}$ concentrations, the $\mathrm{APE}^{+}$strain had a significant fitness advantage compared to APE- Our data suggest that $A P E_{E c}$ expression can protect bacteria against acute ROS stress, such as from an oxidative burst.

45 Given that in the context of infection ROS and RNS are both important mediators of immune response, we tested if APE producing bacteria were also protected from nitric oxide (NO) exposure. Bacteria from mid-log liquid cultures were dosed with NO donating NONOate 
compounds under aerobic conditions (Fig. 5B, C). Dilutions of the reaction mixtures were plated to count colony forming units and percent survival was calculated relative to the phosphate buffered saline (PBS) control incubated without NONOate. We did not observe a significant survival difference upon challenge with spermine NONOate (Fig. 5B). However, the APE ${ }^{+}$strain had a notable decrease in survival relative to the APE- strain when challenged with a supraphysiological level of 50 mM DETA NONOate (Fig. 5C). Granted, there was no discernable difference between $\mathrm{APE}^{+}$and $\mathrm{APE}^{-}$strains at the lower concentration. Taken together, these data suggest that contrary to the ROS assay, expression of $A P E_{E c}$ does not confer a survival advantage to NO exposure.

\section{Expression of the $A P E_{E c} B G C$ induces biofilm formation}

We observed that $\mathrm{APE}^{+}$bacteria form aggregates when grown in liquid culture and we hypothesized that the presence of $A P E_{E c}$ in the outer membrane affects biofilm formation. To test this, we assessed the biofilm formation capacity of $E$. coli $\mathrm{APE}^{-}$and $\mathrm{APE}^{+}$in an in vitro biofilm assay. The individual strains were grown in non-treated, flat-bottom plates for 48 hours and subsequently stained with crystal violet and quantified by measuring optical absorbance levels at $595 \mathrm{~nm}$. We observed a marked increase in biofilm formation for the APE ${ }^{+}$strain (Fig. 6). Next, we set out to determine the contribution to biofilm formation for the individual genes within the $\mathrm{APE}_{\mathrm{Ec}} \mathrm{BGC}$. Only the $\triangle a p e E(\mathrm{ACP})$ and $\triangle a p e N$ (LolB-like protein) strains showed a significant increase in biofilm formation when compared to APE (Fig. S4). Since APE $E_{\mathrm{Ec}}$ biosynthesis is completely abolished in $\triangle a p e E$ (Fig. S3), these data suggest that biofilm formation is not directly caused by APE biosynthesis. Instead, APE expression might cause alterations in regulatory cascades or cell envelope composition that result in increased biofilm formation.

To further characterize the biofilm structure and formation process over time, we visualized the biofilms at 48 and 72 hours using confocal fluorescence microscopy. This confirmed that the APE ${ }^{+}$ strain forms a robust and homogenous biofilm after 48 hours, in comparison to the disperse attachment observed for APE- (Fig. 7A). However, after 72 hours, there is no discernable difference between $\mathrm{APE}^{+}$and $\mathrm{APE}^{-}$strains by confocal microscopy. In order to further investigate this finding, we acquired z-stacked images to assess the homogeneity and density of the respective biofilms. Three-dimensional rendering of these images shows that $A \mathrm{E}_{\mathrm{Ec}}$ expression results in a much thicker biofilm of $\sim 60 \mu \mathrm{m}$, compared to the $\sim 29 \mu \mathrm{m}$ thick APE- aggregates (Fig. 7B). At 72 hours, the APE- biofilm becomes more homogenous in structure, but at $\sim 46 \mu \mathrm{m}$ remains thinner than the denser $\sim 53 \mu \mathrm{m} \mathrm{APE}{ }^{+}$biofilm. These observations were further quantified over time and showed that at 24 and 48 hours, $\mathrm{APE}^{+}$biofilms are on average 2.5 times more voluminous than the APE counterparts (Fig. 7C). In conclusion, APE $\mathrm{Ec}_{\mathrm{c}}$ expression increases the rate of $E$. coli adherence and biofilm formation, resulting in a thicker and denser structure.

\section{DISCUSSION}

We report the first genetic characterization and functional analysis of an APE BGC from a medically relevant bacterial uropathogen. The core biosynthesis for APExd, the product of a related $B G C$ from the nematode symbiont $X$. doucetiae, was elucidated in a recent herculean in vitro effort by Grammbitter et al. [11]. Nine enzymes from the APE $x_{d} B G C$ (ApeC, E, F, H, I, O, P, $Q$ and $R$ ) were produced heterologously in $E$. coli and several of these were found to form stable heterocomplexes. The biosynthetic roles for these enzymes were characterized and the formation of $\mathrm{APE}_{\mathrm{Xd}_{\mathrm{d}}} \mathrm{ACP}$ was reconstituted in vitro. Our genetic analysis of the $E$. coli CFT073 APE $\mathrm{Ec}_{\mathrm{Ec}} \mathrm{BGC}$ complements this biochemical analysis and contributes important in vivo insights into the APE 
biosynthetic process, including the identification of functionally redundant core biosynthetic genes, as well as non-essential genes for cell envelope attachment and transport later in the pathway.

Among the mutants in the core biosynthetic genes, $\triangle a p e E$ is $\mathrm{APE}^{-}$, while $\triangle a p e F$ retains partial pigmentation (Fig. 2), indicating that this latter ACP might be involved in malonyl chain extension and that the deletion could be partially complemented by the ACP involved in E. coli fatty acid biosynthesis, AcpP [30]. This also suggests that ApeF does not load 4-HBA in vivo, making ApeE the preferred ACP used for loading the 4-HBA starter (Figs. 3, 4). This observation is in line with the prior in vitro reconstitution of the respective APExd pathway, which showed that both ACPs could be loaded with 4-HBA, albeit at a much lower efficiency for ApeF [11]. Our deletion analysis indicates that this does not occur at sufficiently high levels for in vivo pathway activity. Moreover, the functional redundancy of ApeF is further illustrated by the observation that not all APE BGCs have a second ACP as is the case for xanthomonadin APE BGCs from Xanthomonas spp. [1, 12]. Deletion of apeK, the gene encoding a putative thioesterase, resulted only in partial loss of pigmentation. No in vitro hydrolysis of 4-HBA-CoA or ACP-APE intermediates was observed for this enzyme, and a proofreading activity for ACP loading was proposed [11]. Since APE $\mathrm{Ec}^{-}$ carboxylic acid production wasn't abolished in vivo, this suggests that the putative proofreading activity is not absolutely required for biosynthesis but could have an impact on yield.

Gram-negative lipid biosynthesis requires adenosine triphosphate (ATP) and therefore typically takes place at the inner leaflet of the cell membrane. Lipids that are destined for the outer membrane are subsequently transported across the periplasm by dedicated transport systems [27]. Several of the genes predicted to be involved in APE $E_{E c}$ cell envelope attachment (apeD, apeJ and apeK) still produce reduced pigmentation due to accumulated intermediates upon knockout, suggesting the biosynthesis of the core APE $E_{E c}$ moiety is not disturbed (Figs. 2, 4). We propose that $A P E_{E c}$ is transferred to an anchor molecule by ApeD (lysophospholipid acyltransferase) and ApeJ (a glycosyltransferase / acyltransferase). This anchoring might be facilitated by ApeK thioesterase-mediated release of $A P E_{E c}$ from its $A C P$. The APE $E_{E c}$-lipid might be transported across the cytoplasmic membrane by ApeM (an RND family transporter, Fig. 8). ApeG is a membrane protein with a predicted COG4648 pyrophosphatase domain that could be involved in providing the energy to drive transport through ATP hydrolysis, in a similar fashion to the role of SecA during protein translocation. Gram-negative bacteria shuttle lipids across the periplasmic space when their hydrophobic acyl chains are shielded from the aqueous milieu. This shielding can happen either via vesicular trafficking or via a protein-assisted process [27]. Since ApeL and ApeN share weak homology to components of the 'localization of lipoprotein' (Lol) pathway and ApeA encodes for a lipoprotein, these proteins could be involved in localization and insertion of the $A P E_{E c}-$ lipid to the outer membrane [27, 31]. ApeGLMN homologs are conserved across different APE BGCs [1], suggesting they constitute dedicated transport machinery with low similarity to currently characterized translocation systems. At present, the identity of the molecule that covalently anchors $A P E_{E c}$ in the outer membrane is still not known. Conserved domains in the two acyltransferases ApeD and ApeJ suggests that this molecular anchor could be a glycolipid, potentially lipid A or a derivative thereof. Alternatively, the presence of a predicted lipoprotein and Lol-like transport genes could imply that APEs might get localized to the outer membrane while serving as a lipid anchor for ApeA. During the revision of this manuscript, Grammbitter et al. solved the structure of the APE-containing lipid from $X$. doucetiae [32]. It is feasible that the APE $E_{E C}$ is attached to a similar type of lipid in the E. coli cell envelope.

The generation of ROS constitutes a critical component of the initial line of defense by phagocytes against invading bacterial pathogens [20]. In turn, bacteria have developed several mechanisms to protect themselves from ROS damage. Enzymatic detoxification is an important mechanism employed to deal with an intracellular excess of ROS [33]. Three types of enzymes are key in this 
process: superoxide dismutases catalyze the dismutation of superoxide into $\mathrm{O}_{2}$ and $\mathrm{H}_{2} \mathrm{O}_{2}$, and catalases and peroxiredoxins detoxify $\mathrm{H}_{2} \mathrm{O}_{2}$ by enzymatic degradation [21]. As a complementary strategy, cells express polyenes in their envelope to form a protective shield that quenches ROS, thereby preventing their entry into the cell $[18,29]$. One of the most well-known groups of pigments with a polyene chromophore that display antioxidant activity are the carotenoids. While their functional analogy to APEs in bacterial protection from ROS has been recognized [1, 4], carotenoids are produced by a biosynthetically distinct process. These lipophilic isoprenoids are assembled from isopentenyl pyrophosphate and dimethylallyl pyrophosphate building blocks by the subsequent action of a prenyl transferase, phytoene synthase and phytoene desaturase [34]. Examples of bacterial carotenoids that protect their producers from external ROS are the 'golden' pigment staphyloxanthin, produced by $S$. aureus (Fig. 1) and the red pigment deinoxanthin from Deinococcus radiodurans [18, 29, 35]. Amide-containing polyenes are a different family of pigments that are present in Gram-positive cell envelopes. These compounds are produced by a fatty-acid-like type II polyketide pathway more closely related to APE biosynthesis. The red amidecontaining pigment granadaene is an ornithine rhamno-polyene produced by group B Streptococcus and it has been implicated in oxidative stress protection (Fig. 1, [17, 19]). A related amide-containing polyene was isolated from Propionibacterium jensenii [36] and a related BGC from commensal Lactobacillus reuteri was shown to produce a polyenic small molecule that stimulates the host aryl hydrocarbon receptor [37]. A potential role in bacterial protection from

20 ROS remains to be investigated for the latter two examples. We challenged $A P E_{E c}$ expressing bacteria with various concentrations of $\mathrm{H}_{2} \mathrm{O}_{2}$ and found that they significantly increased survival compared to the control strain (Fig. 5). Our results are corroborated by other studies showing that APEs protect from oxidative stress in a variety of different bacterial genera, including Variovorax [4], Lysobacter [6] and Xanthomonas [38-40]. Carotenoids and amide-containing polyenes present an interesting example of convergent evolution in Gram-positive bacteria and our results suggest an analogous function for APEs in Gram-negative bacteria.

Contrary to their ROS protective function, we did not observe a discernable survival advantage of $\mathrm{APE}_{\mathrm{Ec}}$ expression upon RNS challenge. The differential susceptibility to $50 \mathrm{mM}$ DETA-NONOate suggests more investigation is needed to understand if APE expression compromises respiratory metabolism flexibility and how this might impact $E$. col's competitive advantage in intestinal colonization [41]. Alternatively, our observation might be an artifact of the aerobic environment under which this experiment was completed. In addition, E. coli expresses a number RNS mediators such as flavohemoprotein ( $\mathrm{Hmp}$ ) which primarily functions in aerobic conditions, and flavorubredoxin (NorVW) and cytochrome c nitrate reductase (NrfA) which are only active under anaerobic or microoxic conditions [42]. These additional defenses may overshadow the contribution of aryl polyenes. In the context of infection where the phagosome enclosed bacteria will encounter both $\mathrm{H}_{2} \mathrm{O}_{2}$ and $\mathrm{NO}$ after the macrophage has activated iNOS, there is a prioritization of $\mathrm{H}_{2} \mathrm{O}_{2}$ mitigation by $E$. coli [43]. Whether aryl polyenes might provide some beneficial effect during acute exposure to ROS and RNS together may be better studied using an in vitro model.

40 Laboratory strains of E. coli K-12 are typically poor biofilm formers [44]. It was therefore surprising that heterologous expression of the APE $E_{E C}$ BGC from the UPEC strain CFT073 in Top10 resulted in a robust increase in biofilm formation. There are various cell-envelope associated factors that contribute to $E$. coli adhesion to solid surfaces and biofilm formation, including type 1 and curli fimbriae, conjugative pili, autotransporters, cell surface polysaccharides, and capsules [44-46]. The mechanism by which $A P E_{E c}$ expression contributes to the biofilm formation process remains to be determined. It is possible that APE expression contributes indirectly by activating regulatory cascades involved in biofilm formation. However, since APEs are likely part of a larger glycolipid molecule, it is feasible that the cell surface localization of this molecular entity structurally supports the polysaccharide matrix. Alternatively, APE expression could cause a change of the outer 
membrane that results in increased exposure of previously characterized cell-surface adhesion factors.

Uropathogenic E. coli, such as the APE $\mathrm{Ec}_{\mathrm{c}}$ parent strain CFT073, display a biofilm-like phenotype during urinary tract infection (UTI) [47]. After colonization of the urethra, bacteria ascend to the bladder where they can invade epithelial cells. The intracellular bacteria then replicate rapidly and form biofilm-like intracellular bacterial communities that can remain stable for multiple weeks, even after a standard course of oral antibiotic treatment has been completed. These bacteria can flux out of the epithelial cells in large numbers and cause the infection to resurge, and potentially ascend to the kidneys, thereby causing a more severe disease state such as pyelonephritis. The partly intracellular lifestyle of UPEC makes treatment challenging, since antibiotics cannot penetrate into the epithelial cells and the treatment regimen might be shorter than the time the bacteria persist intracellularly [48]. In addition, biofilm formation on indwelling devices, such as stents, implants, and catheters can form reservoirs of pathogenic bacteria thus leading to chronic infections. Catheter-associated urinary tract infections are the most common hospital acquired infections in the USA, and UPEC strains are the predominant cause [49]. Interestingly, overexpression of the transcriptional regulator TosR in E. coli CFT073 caused increased biofilm formation, as well as induced expression of the APE $E_{\mathrm{EC}} \mathrm{BGC}$ [50], though no functional link between these two events has yet been established. Our future studies will focus on the antioxidant and biofilm-inducing roles of APEs in the context of in vivo bacterial pathogenesis.

Several examples exist within the Enterobacteriaceae, where APE BGCs are located on pathogenicity islands $[1,51,52]$, suggesting they could be transmitted to other bacteria through horizontal gene transfer. This potential mobility is a major concern, especially given their roles in protection from oxidative stress and biofilm formation. It is therefore important to gain a fundamental understanding of APE biosynthesis and biology, in order to develop APE-targeting strategies for combating multidrug-resistant pathogens and preventing their biofilm formation.

Collectively, our data demonstrate the importance of studying the in vivo production of APEs, highlighting key differences not appreciable using elegant in vitro systems. Moreover, we have demonstrated a potential role for APEs in the pathogenic function of Gram-negative Enterobacteriaceae, mediating resistance to oxidative stress and facilitating robust biofilm formation. Our work provides essential molecular detail that could advance efforts to develop much-need therapeutic strategies to treat pathogenic E. coli.

\section{METHODS}

\section{General methods, bacterial cultures and growth media}

E. coli cultures were grown as liquid or solid agar media, using a Luria-Bertani (LB) or MuellerHinton base. Incubation was at $37^{\circ} \mathrm{C}$, except for the strains carrying a temperature sensitive ori plasmid that required $28^{\circ} \mathrm{C}$ incubation. Antibiotic concentrations used for selection and maintenance were carbenicillin $(100 \mu \mathrm{g} / \mathrm{ml})$, kanamycin $(50 \mu \mathrm{g} / \mathrm{ml})$ and apramycin $(50 \mu \mathrm{g} / \mathrm{ml})$. Plasmid DNA isolation was performed with a standard kit according to the manufacturer's instructions (Qiagen). General microbiological manipulations, PCR amplification, cloning and transformation procedures were as described previously [53]. Enzymes were purchased from New England Biolabs, and chemicals from MilliporeSigma or Fisher Scientific, unless otherwise stated. Oligonucleotide primers were obtained from Integrated DNA Technologies. Statistical analyses were performed as indicated in each figure legend, using GraphPad Prism version 9.0.0 (GraphPad Software). 


\section{In-frame scarred mutant construction}

The bacterial strains, primers and plasmids used or generated in this study are listed in Tables $\mathrm{S} 1-3$. Individual genes in the $\mathrm{APE} \mathrm{E}_{\mathrm{Ec}} \mathrm{BGC}$ were replaced with an $81 \mathrm{bp}$ in-frame scar sequence in a two-step PCR-generated recombineering process [28]. The procedure is illustrated

5 schematically in Fig. S1 for apeK, other genes were replaced in an analogous manner. pJC121 is the parent plasmid that was used for generating all $\Delta$ scar derivatives ([1], Table S3). Briefly, in the first step, a targeting cassette is generated by PCR amplification of the FRT sites that flank the apramycin resistance gene from plJ773. The primers used for amplification contain large (on average $58 \mathrm{nt}$ ) 5 ' tails that are homologous to the up and downstream regions of the gene to be deleted (Table S2). The plJ121 parent vector and targeting cassette are introduced into E. coli BW25113 with plJ790 (a ARED recombination plasmid) and the genes of interest are replaced with the apra $^{R}$ marker via double homologous recombination, yielding the respective $\Delta$ apra ${ }^{R}$ constructs for each gene (even numbered plasmids pJC130-164 in Table S3). In the second step, the resistance markers are removed by introducing the $\Delta$ apra $^{R}$ plasmids into E. coli BT340. Expression of Flp recombinase by this strain causes recombination of the FRT sites that flank the apra $^{\mathrm{R}}$ marker, leaving an 81 bp scar sequence. The resulting in-frame scarred mutant plasmids can be identified based on their apramycin sensitivity (odd numbered plasmids PJC131-165 in Table S3).

\section{Analytical profiling of APE-related metabolites}

A single colony of the $\mathrm{APE}^{-}, \mathrm{APE}^{+}$and different $\triangle$ ape $E$. coli strains was used to inoculate $50 \mathrm{ml}$ liquid cultures in LB + kanamycin $(50 \mu \mathrm{g} / \mathrm{ml})$. Liquid cultures were incubated for 24 hours at $37^{\circ} \mathrm{C}$ ( $250 \mathrm{rpm}$, in the dark) prior to harvesting the cell pellets by centrifugation ( $3500 \mathrm{~g}, 10 \mathrm{~min}$ ). The growth medium was decanted and the cell pellets were washed twice by resuspension in $\mathrm{dH}_{2} \mathrm{O}$ followed by centrifugation. Cell pellets were extracted once with $25 \mathrm{ml}$ acetone and then twice with $25 \mathrm{ml}$ acetone:methanol (2:1 vol/vol). The extracts were filtered over paper and combined. Next, organic solvent was removed by a rotary evaporator. The dried samples were reconstituted in $\mathrm{MeOH}$ and passaged over a silica column prior to HPLC analysis. HPLC conditions used a gradient of acetonitrile in $0.1 \%$ trifluoroacetic acid water: Initiating with a hold at $100 \%$ water for 3 min, followed by gradual increase from $0 \%$ to $100 \%$ ACN between $3 \mathrm{~min}$ and $23 \mathrm{~min}$, and ending in a $100 \%$ ACN hold for 3 minutes, followed by a 1 min re-equilibration to $100 \%$ aqueous phase and $3 \mathrm{~min}$ hold at $100 \%$ aqueous. Detection was at $\lambda=441 \mathrm{~nm}$.

\section{$\mathrm{H}_{2} \mathrm{O}_{2}$ survival assay}

For $\mathrm{H}_{2} \mathrm{O}_{2}$ survival assays, a starter culture was grown overnight in $\mathrm{LB}$, cells were pelleted and resuspended in PBS at $\mathrm{OD}_{600}=1$. Equal volumes of $\mathrm{H}_{2} \mathrm{O}_{2}$ (in PBS) were added with final concentrations ranging from 0 to $7 \mathrm{mM}$. Reactions were incubated at room temperature for 1 hour, quenched by addition of excess catalase and serially diluted prior to plating onto LB agar. Colony forming units were counted after overnight incubation at $37^{\circ} \mathrm{C}$ and percent survival was calculated relative to the PBS only control.

\section{NONOate survival assays}

For the NONOate survival assays, a starter culture was grown for $4 \mathrm{hrs}$ at $37^{\circ} \mathrm{C}, 220 \mathrm{rpm}$. The cultures with similar starting OD readings were spun down and washed with PBS. The pellet was 
resuspended in PBS to a final OD600 of 0.3. Equal volumes of DETA NONOate (Cayman, 82120) or spermine NONOate (Cayman, 82150) were diluted in PBS and added to the bacteria. The final concentrations of the reactions were 25 and $50 \mathrm{mM}$ for DETA NONOate, and 0.5 or $1 \mathrm{mM}$ for spermine NONOate with PBS controls. Reactions were incubated for 1 hour (DETA NONOate) or 2 hours (spermine NONOate) and serially diluted prior to plating onto LB agar. Colony forming units were counted after overnight incubation at $37^{\circ} \mathrm{C}$ and percent survival was calculated relative to the PBS only control.

\section{E. coli biofilm assays}

An overnight culture of $\mathrm{APE}^{+}$and $\mathrm{APE}^{-}$E. coli was adjusted to $\mathrm{OD}_{600}=1$, and $10 \mu$ was used to inoculate a total of $1 \mathrm{ml}$ fresh medium in flat-bottom, non-tissue culture treated plates. Biofilms were grown by static incubation at $37^{\circ} \mathrm{C}$ for 24,48 or 72 hours. All steps were performed with care to not disturb the biofilms. After removing the supernatant, cells were fixed for 30 minutes with $250 \mu \mathrm{l}$ Bouin's solution (Sigma). The fixing solution was removed and cells were washed twice with $250 \mu \mathrm{l}$ sterile $\mathrm{H}_{2} \mathrm{O}$ prior to staining for quantification or confocal imaging. For quantitative staining, fixed samples were submerged in $250 \mu \mathrm{l}$ of a $0.1 \% \mathrm{v} / \mathrm{v}$ crystal violet solution for 15 minutes. The staining solution was removed and samples were washed twice with $250 \mu$ sterile $\mathrm{H}_{2} \mathrm{O}$. The remaining material was dissolved in $300 \mu \mathrm{l}$ of $95 \% \mathrm{v} / \mathrm{v}$ ethanol, and quantified by measuring absorbance at $595 \mathrm{~nm}$. Fixed biofilm samples that were processed for confocal imaging were stained with $250 \mu \mathrm{l}$ of SYTO 9 green fluorescent dye. Confocal laser scanning microscopy image acquisition was performed using a Leica TCS SP5, using the 20x objective. Volocity (Quorum Technologies) was used to measure biofilm thickness and to produce threedimensional rendered images by combining z-stacked images that were recorded at a z-step of $0.25 \mu \mathrm{m}$. Biofilm volumes were calculated by using the Volocity measurements and the software's "find object" features.

\section{ACKNOWLEDGEMENTS}

We thank Dr. John W Peterson and Dr. Judith A Drazba of the LRI imaging core for assistance with the biofilm imaging. Funding: JC is supported by seed funding from the Cleveland Clinic 30 Foundation, a Research Grant from the Prevent Cancer Foundation (PCF2019-JC), an American Cancer Society Institutional Research Grant (IRG-16-186-21) and a Jump Start Award (CA043703) from the Case Comprehensive Cancer Center. This work was supported in part by National Institutes of Health grants R01 DK120679 (JMB), P50 AA024333 (JMB), and P01 HL147823 (JMB). Author contributions: Conceptualization and design: IJ, JC. Investigation: IJ, LJO, RLM, EAM, AK, KBS, NN, JC. Figures: IJ, LJO, RLM, JC. Writing of the original draft: IJ, JC. Writing, review and interpretation: IJ, LJO, RLM, PPA, JMB, JC. Competing interests: none declared. Materials and correspondence: Please address all correspondence and material requests to JC at claesej@ccf.org 


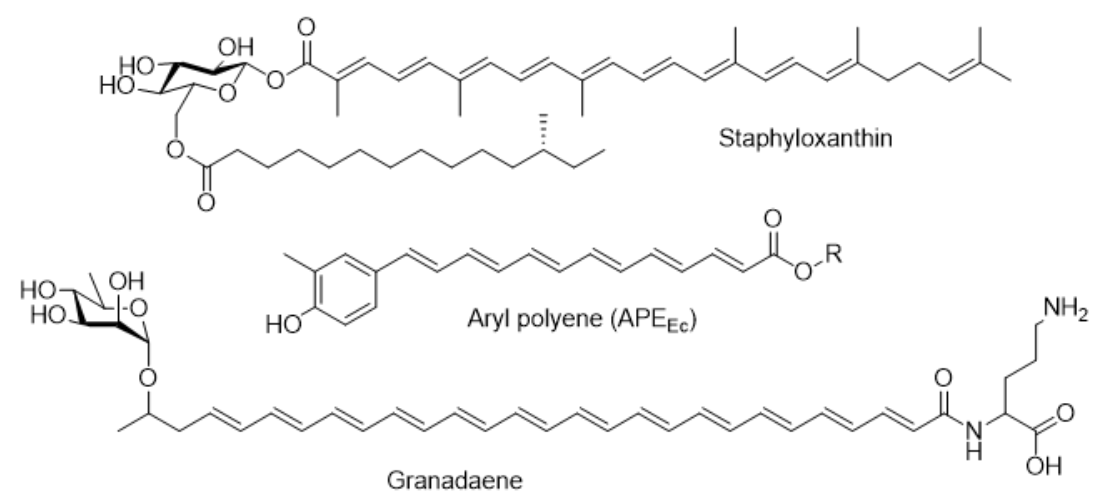

Figure 1. Chemical structures of pigmented fitness factors localized in the cell envelope of bacterial pathogens. Staphyloxanthin is the carotenoid produced by $S$. aureus responsible for the organism's characteristic golden color (top). Aryl polyenes are widespread yellow pigments among Gram-negative pathogens (middle), for example $\mathrm{APE}_{\mathrm{Ec}}$ from uropathogenic $E$. coli. Granadaene is an ornithine rhamno-polyene that constitutes the red pigmentation in Group B Streptococcus (bottom) [1, 16, 17]. 


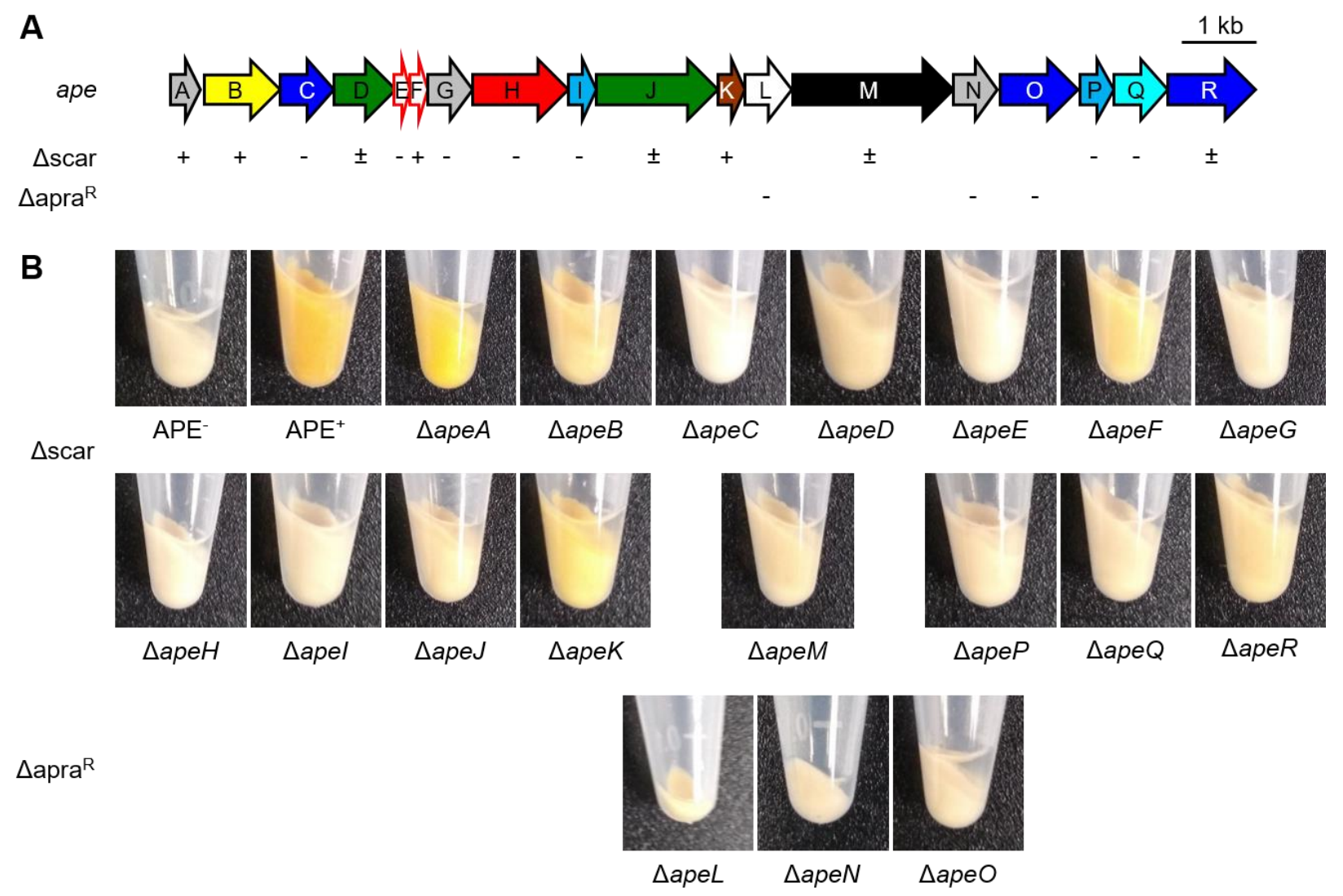

Figure 2. Pigmentation phenotype of individual deletion mutants in the $A P E_{E c} B G C$. A) Schematic arrow representation of the E. coli CFT073 APE ${ }_{\mathrm{Ec}} \mathrm{BGC}$ (see Table 1 for predicted gene functions). The pigmentation phenotype of individual scarred deletion mutants ( $\Delta$ scar) is reported below the BGC as follows: + (near wild-type level pigmentation), \pm (largely reduced pigmentation), - (non-pigmented). We did not obtain scarred deletion mutants for apaL, apeN and apeO, and their respective $\Delta \mathrm{apra}^{\mathrm{R}}$ mutants are not pigmented. B) Pigmentation phenotypes of the cell pellets from individual $\triangle$ ape strains in comparison to cell pellet pigmentation from APEand $\mathrm{APE}^{+}$cultures. The cell pellet extracts (reconstituted in $\mathrm{MeOH}$ ) of these strains are depicted in Fig. S2. 


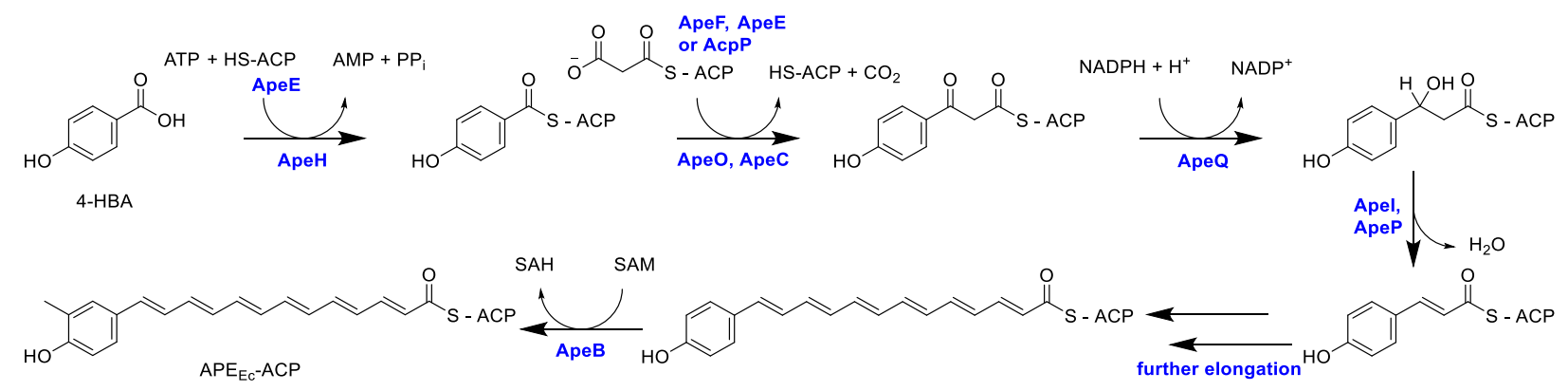

Figure 3. Proposed pathway for $A P E_{E c}$ carboxylic acid biosynthesis. $A P E_{E c}$ biosynthesis is predicted to start with the ACP-activation of 4-hydroxybenzoic acid (4-HBA), an early intermediate in ubiquinone biosynthesis. The aromatic starter is further elongated with malonyl-ACP units (potentially carried by ApeF, ApeE, or the ACP involved in fatty acid biosynthesis, AcpP [30]) by repetitive action of fatty-acid-like type II polyketide biosynthetic enzymes: ketosynthase ApeO and ketosynthase-chain length factor ApeC, ketoreductase ApeQ and dehydratases Apel and/or ApeP. The SAM-dependent methyltransferase ApeB is involved in methylation of the aromatic head group, and it remains to be determined whether this modification occurs before or after ApeK

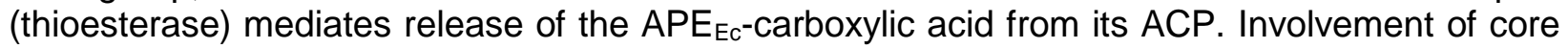
enzymes in the pathway are indicated for E. coli CFT073 (in blue). SAM = S-adenosyl-Lmethionine, SAH = S-adenosyl-L-homocysteine. 


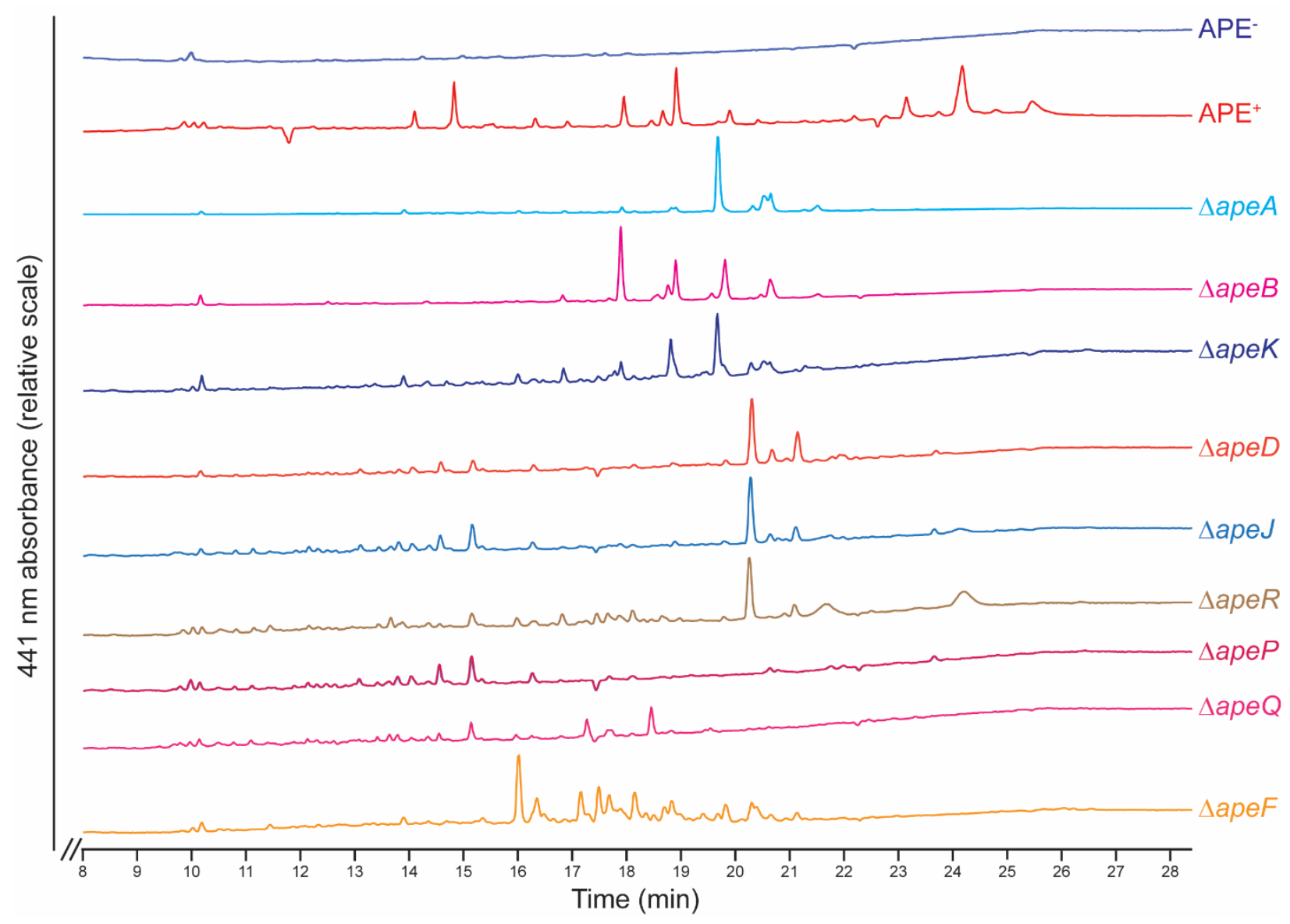

Figure 4. HPLC traces of $\boldsymbol{E}$. coli $\Delta$ ape strain crude extracts. The $\mathrm{APE}^{+}$extract (red) contained three clusters of APE-related peaks (around $14.5 \mathrm{~min}(57.5 \%$ acetonitrile (ACN)), $19 \mathrm{~min}(80 \%$ ACN) and 24 min (100\% ACN)) which are not present in the APE strain (dark blue). The primary APE lipid eluting at $100 \%$ ACN only occurs in the $\mathrm{APE}^{+}$and $\triangle a p e R$ extracts. The $\triangle a p e A, \triangle a p e B$ and $\triangle a p e K$ strains produce a comparable set of immature APE carboxylic acids (early intermediates prior to initiation of the attachment process). $\Delta a p e D, \Delta a p e J$ and $\Delta a p e R$ produce late pathway intermediates, which likely consist of APE carboxylic acids that are impaired in their proper attachment to the final outer membrane lipid. Deletion of the apeF ACP (orange) results in the formation of a series of yellow pigmented intermediates, indicating it is not strictly required for biosynthesis of the APE carboxylic acid moiety. HPLC traces for all $\triangle$ ape strains that did not have significant $441 \mathrm{~nm}$ absorbance are shown in Fig. S3. HPLC conditions used a gradient of ACN in $0.1 \%$ trifluoroacetic acid (TFA) water, initiating with a hold at $100 \%$ water for $3 \mathrm{~min}$, followed by gradual increase from $0 \%$ to $100 \%$ ACN between 3 min and 23 min, and ending in a $100 \%$ ACN hold. Detection was at $\lambda=441 \mathrm{~nm}$. 

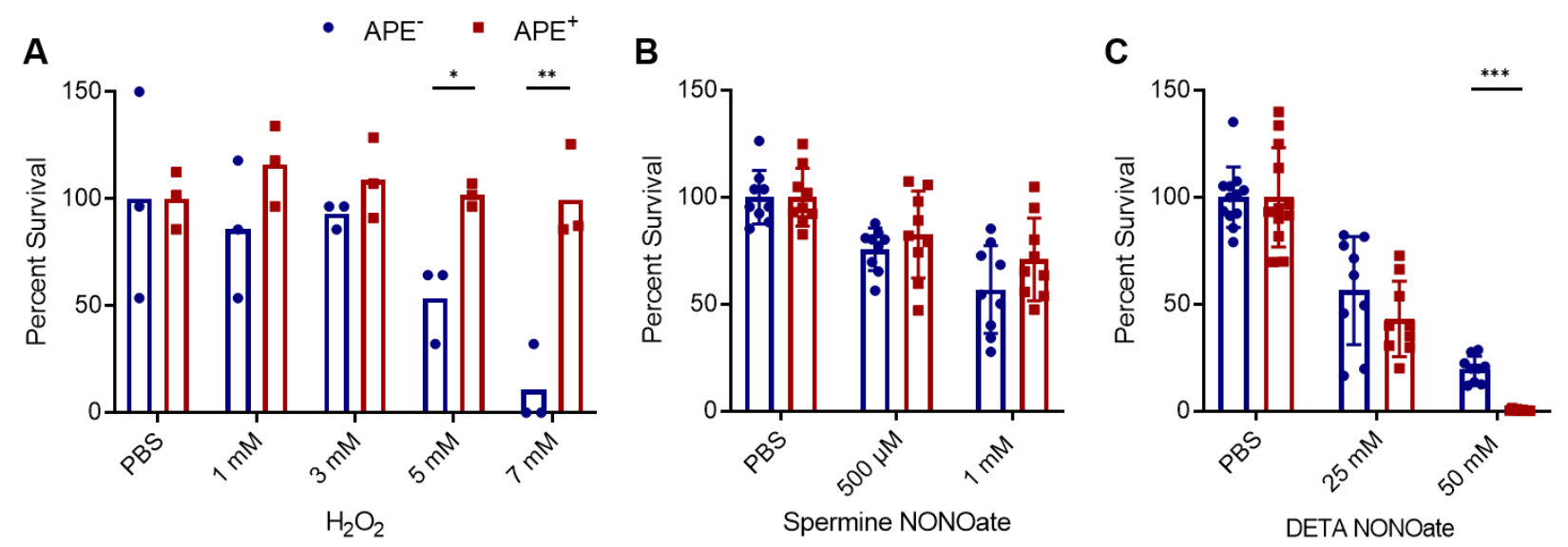

Figure 5. $A P E_{E c}$ expression protects from oxidative, but not from nitrosative challenge. A) Percent survival upon exposure to different concentrations of hydrogen peroxide $\left(\mathrm{H}_{2} \mathrm{O}_{2}\right)$ is calculated from colony forming units after a 60 -minute $\mathrm{H}_{2} \mathrm{O}_{2}$ challenge for the APE $\mathrm{E}_{\mathrm{Ec}}$ expressing $\left(\mathrm{APE}^{+}\right.$, red) versus control strain ( $\mathrm{APE}^{-}$, blue) relative to the PBS control. The reactions were stopped by adding an excess of catalase. B) APE $E_{E c}$ expression does not mediate protection from reactive nitrogen challenge. Percent survival is calculated from colony forming units after a $1-2$ hour spermine NONOate challenge for the $\mathrm{APE}_{\mathrm{Ec}}$ expressing $\left(\mathrm{APE}^{+}\right)$versus control strain (APE') relative to the PBS control. C) Percent survival of $\mathrm{APE}^{+}$and $\mathrm{APE}^{-}$strains after 1-2 hours of DETA

NONOate challenge. PBS = phosphate buffered saline $(n=3-5$ repeats; error bars represent SD; ${ }^{*} p<0.05,{ }^{* *} p<0.01$ and ${ }^{* *} p<0.001$ by unpaired t-test with Welch's correction). 


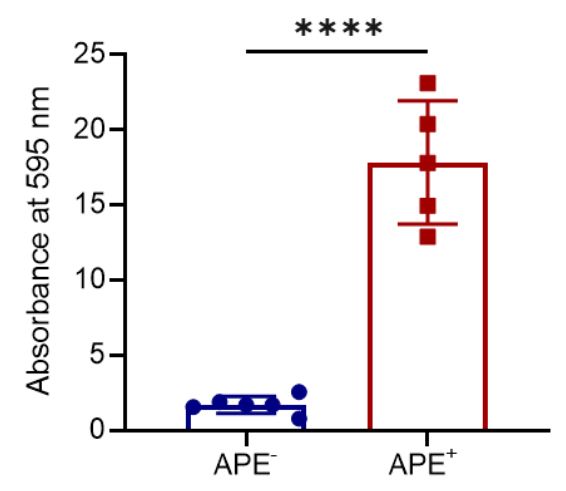

Figure 6. APE $E_{\mathrm{Ec}}$ expression increases E. coli biofilm formation. Biofilms were grown in nontreated multi-well plates for 48 hours and subsequently stained with crystal violet. Absorbance at $595 \mathrm{~nm}$ was measured and normalized to APE values ( $\mathrm{n}=3$ repeats; error bars represent SD; ${ }^{* * * *} p<0.0001$ by unpaired, two-tailed t-test). 

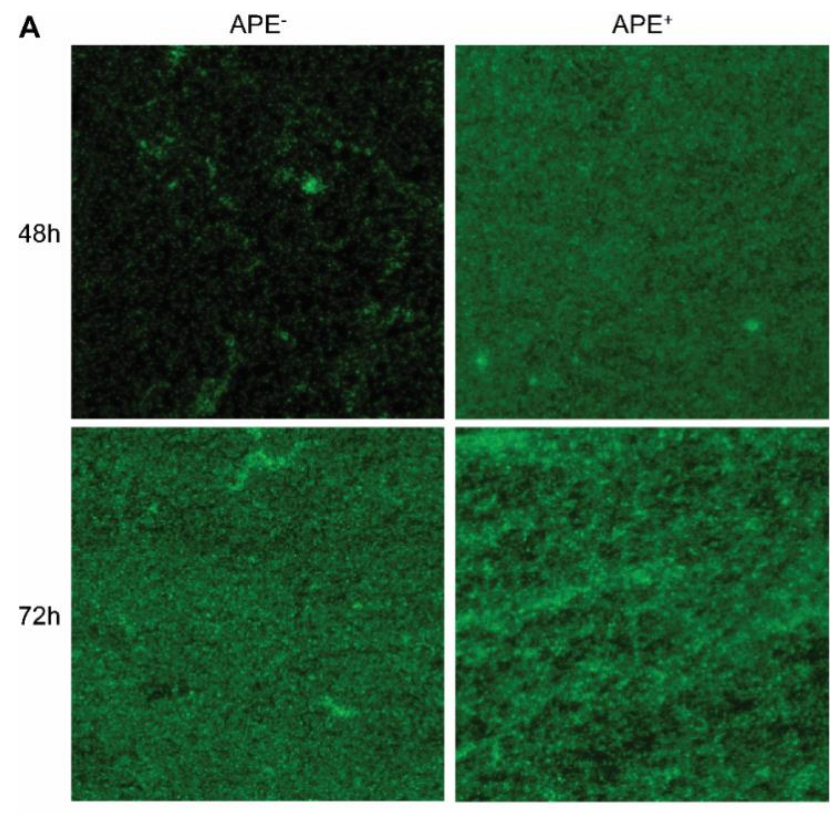

B
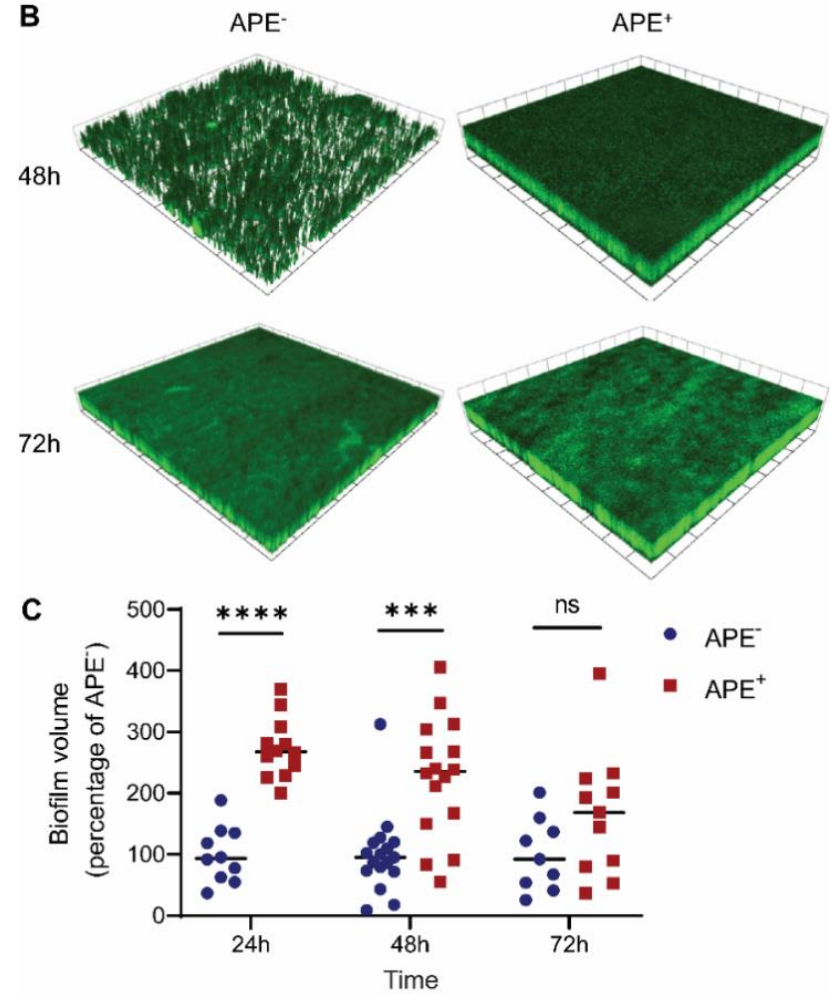

Figure 7. $A P E_{E c}$ expressing E. coli more rapidly form thicker and denser biofilms. A) Confocal fluorescence microscopy images of $\mathrm{APE}^{-}$and $\mathrm{APE}^{+} E$. coli biofilms that were grown in non-tissue culture treated multi-well plates for 48 hours and 72 hours. Samples were fixed, stained with SYTO 9, and imaged using the $63 \times$ objective. B) Three-dimensional rendering of the $z-$ stacked images for the $\mathrm{APE}^{-}$and $\mathrm{APE}^{+}$biofilms shows that $\mathrm{APE}_{\mathrm{Ec}}$ expression results in formation of a thicker and denser biofilm. 1 scale unit $=57.97 \mu \mathrm{m}$. C) The volume of biofilm produced by $\mathrm{APE}^{+}$(red) is on average 2.5 times higher than for APE- (blue) after 24 and 48 hours of growth. Volocity (Quorum Technologies) was used to measure biofilm thickness, render 3D-images and calculate biofilm volumes. ( $\mathrm{n}=3$ repeats; ${ }^{* \star} p<0.001,{ }^{* \star * *} p<0.0001$ by unpaired, two-tailed t-test). 


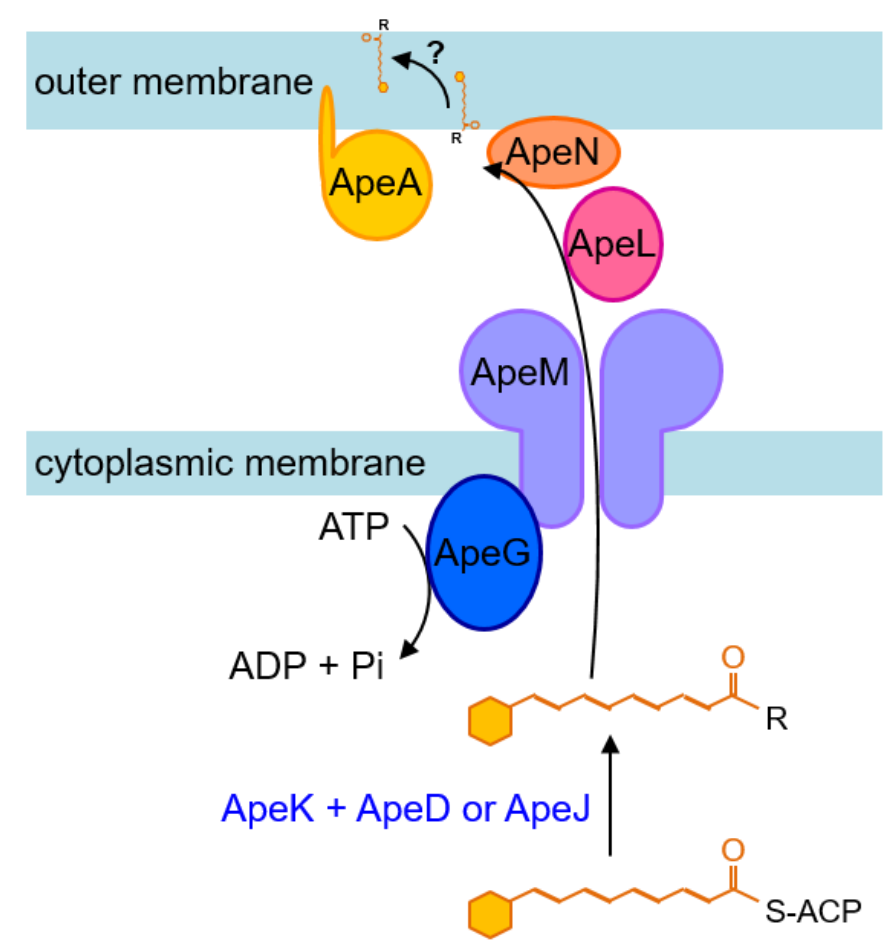

Figure 8. Schematic diagram for APE export and outer membrane localization. Following biosynthesis of the core APE $\mathrm{Ec}_{\mathrm{C}}$-carboxylic acid (Fig. 4), we propose that this entity is attached to a larger lipid anchor molecule $(\mathrm{R})$ by ApeD (lysophospholipid acyltransferase) and ApeJ (a glycosyltransferase / acyltransferase). This attachment process might be assisted by the ApeK thioesterase-mediated release of $A P E_{E c}$ from its $A C P$. The APE $E_{E c}$-lipid might be transported across the cytoplasmic membrane by ApeM (an RND family transporter). The energy for this translocation could be provided by ATP hydrolysis catalyzed by ApeG (a predicted membraneassociated protein with a COG4648 pyrophosphatase domain). Since ApeL and ApeN share weak homology to components of the 'localization of lipoprotein' (Lol) pathway and ApeA encodes for a lipoprotein, these proteins could be involved in localization of the APE $E_{E c}$-lipid to the outer membrane. Finally, the APE $\mathrm{Ec}_{\mathrm{c}}$ containing anchor lipid is potentially flipped to the outer leaflet of the outer membrane. 
Table 1. Predicted gene functions for the $A P E_{E c} B G C$.

\begin{tabular}{lll}
\hline ape & Locus tag & Predicted function \\
\hline $\mathbf{A}$ & c1204 & lipoprotein \\
$\mathbf{B}$ & c1203 & SAM-dependent methyltransferase \\
$\mathbf{C}$ & c1202 & 3-ketoacyl-ACP synthase \\
$\mathbf{D}$ & c1201 & lysophospholipid acyltransferase \\
$\mathbf{E}$ & c1200 & acyl carrier protein \\
$\mathbf{F}$ & c1199 & acyl carrier protein \\
$\mathbf{G}$ & c1198 & COG4648 - pyrophosphatase \\
$\mathbf{H}$ & c1197 & acyl-ACP synthetase \\
$\mathbf{I}$ & c1196 & 3-hydroxyacyl-ACP dehydratase \\
$\mathbf{J}$ & c1194 & glycosyltransferase / acyltransferase \\
$\mathbf{K}$ & c1193 & thioesterase \\
$\mathbf{L}$ & c1192 & LolA-like molecular chaperone \\
$\mathbf{M}$ & c1191 & RND family transporter \\
$\mathbf{N}$ & c1190 & DUF3261-LolB-like membrane protein \\
$\mathbf{O}$ & c1189 & 3-ketoacyl-ACP synthase \\
$\mathbf{P}$ & c1188 & 3-hydroxyacyl-ACP dehydratase \\
$\mathbf{Q}$ & c1187 & 3-ketoacyl-ACP reductase \\
$\mathbf{R}$ & c1186 & 3-ketoacyl-ACP synthase \\
\hline
\end{tabular}




\section{References}

1. Cimermancic, P., et al., Insights into secondary metabolism from a global analysis of prokaryotic biosynthetic gene clusters. Cell, 2014. 158(2): p. 412-21.

2. $\mathrm{Xu}, \mathrm{H}$., et al., Innate immune sensing of bacterial modifications of Rho GTPases by the Pyrin inflammasome. Nature, 2014. 513(7517): p. 237-41.

3. Drevinek, P. and E. Mahenthiralingam, Burkholderia cenocepacia in cystic fibrosis: epidemiology and molecular mechanisms of virulence. Clin Microbiol Infect, 2010. 16(7): p. 821-30.

4. Schoner, T.A., et al., Aryl Polyenes, a Highly Abundant Class of Bacterial Natural Products, Are Functionally Related to Antioxidative Carotenoids. Chembiochem, 2016. 17(3): p. 24753.

5. Reichenbach, H., et al., Flexirubin-type pigments in Flavobacterium. Archives of Microbiology, 1980. 126(3): p. 291-293.

6. Wang, Y., et al., Biosynthetic mechanism for sunscreens of the biocontrol agent Lysobacter enzymogenes. PLoS One, 2013. 8(6): p. e66633.

7. Jenkins, C.L. and M.P. Starr, The pigment ofXanthomonas populi is a nonbrominated arylheptaene belonging to xanthomonadin pigment group 11. Current Microbiology, 1982. 7(4): p. 195-198.

8. Achenbach, H., W. Kohl, and H. Reichenbach, Untersuchungen an Stoffwechselprodukten von Mikroorganismen, XI: Flexirubin, ein neuartiges Pigment aus Flexibacter elegans. Chem Ber, 1976. 109(7): p. 2490-2502.

9. Achenbach, H., W. Kohl, and H. Reichenbach, Zur Struktur des Flexirubins. Tetrahedron Lett, 1974. 30: p. 2555-2556.

10. Andrewes, A.G., et al., Xanthomonas pigments. 2. The Xanthomonas "carotenoids"--noncarotenoid brominated aryl-polyene esters. Acta Chem Scand, 1973. 27(7): p. 2383-95.

11. Grammbitter, G.L.C., et al., An Uncommon Type II PKS Catalyzes Biosynthesis of Aryl Polyene Pigments. J Am Chem Soc, 2019.

12. Goel, A.K., et al., Genetic locus encoding functions involved in biosynthesis and outer membrane localization of xanthomonadin in Xanthomonas oryzae pv. oryzae. J Bacteriol, 2002. 184(13): p. 3539-48.

13. Flemming, H.C., et al., Biofilms: an emergent form of bacterial life. Nat Rev Microbiol, 2016. 14(9): p. 563-75.

14. Costerton, J.W., P.S. Stewart, and E.P. Greenberg, Bacterial biofilms: a common cause of persistent infections. Science, 1999. 284(5418): p. 1318-22.

15. Lewis, K., Riddle of biofilm resistance. Antimicrob Agents Chemother, 2001. 45(4): p. 9991007.

16. Pelz, A., et al., Structure and biosynthesis of staphyloxanthin from Staphylococcus aureus. J Biol Chem, 2005. 280(37): p. 32493-8.

17. Rosa-Fraile, M., et al., Granadaene: proposed structure of the group B Streptococcus polyenic pigment. Appl Environ Microbiol, 2006. 72(9): p. 6367-70.

18. Clauditz, A., et al., Staphyloxanthin plays a role in the fitness of Staphylococcus aureus and its ability to cope with oxidative stress. Infect Immun, 2006. 74(8): p. 4950-3.

19. Liu, G.Y., et al., Sword and shield: linked group B streptococcal beta-hemolysin/cytolysin and carotenoid pigment function to subvert host phagocyte defense. Proc Natl Acad Sci U S A, 2004. 101(40): p. 14491-6.

20. Forman, H.J. and M. Torres, Reactive oxygen species and cell signaling: respiratory burst in macrophage signaling. Am J Respir Crit Care Med, 2002. 166(12 Pt 2): p. S4-8.

21. Fang, F.C., Antimicrobial actions of reactive oxygen species. mBio, 2011. 2(5).

22. Fang, F.C., Antimicrobial reactive oxygen and nitrogen species: concepts and controversies. Nat Rev Microbiol, 2004. 2(10): p. 820-32. 
23. Liu, C.I., et al., A cholesterol biosynthesis inhibitor blocks Staphylococcus aureus virulence. Science, 2008. 319(5868): p. 1391-4.

24. Nichols, B.P. and J.M. Green, Cloning and sequencing of Escherichia coli ubiC and purification of chorismate lyase. J Bacteriol, 1992. 174(16): p. 5309-16.

25. De Lay, N.R. and J.E. Cronan, A genome rearrangement has orphaned the Escherichia coli K-12 AcpT phosphopantetheinyl transferase from its cognate Escherichia coli O157:H7 substrates. Mol Microbiol, 2006. 61(1): p. 232-42.

26. Nikaido, H., RND transporters in the living world. Res Microbiol, 2018. 169(7-8): p. 363-371.

27. Powers, M.J. and M.S. Trent, Intermembrane transport: Glycerophospholipid homeostasis of the Gram-negative cell envelope. Proc Natl Acad Sci U S A, 2019. 116(35): p. 1714717155.

28. Gust, B., et al., PCR-targeted Streptomyces gene replacement identifies a protein domain needed for biosynthesis of the sesquiterpene soil odor geosmin. Proc Natl Acad Sci U S A, 2003. 100(4): p. 1541-6.

29. Liu, G.Y., et al., Staphylococcus aureus golden pigment impairs neutrophil killing and promotes virulence through its antioxidant activity. J Exp Med, 2005. 202(2): p. 209-15.

30. De Lay, N.R. and J.E. Cronan, In vivo functional analyses of the type II acyl carrier proteins of fatty acid biosynthesis. J Biol Chem, 2007. 282(28): p. 20319-28.

31. Konovalova, A. and T.J. Silhavy, Outer membrane lipoprotein biogenesis: Lol is not the end. Philos Trans R Soc Lond B Biol Sci, 2015. 370(1679).

32. Grammbitter, G.L.C., et al., The Chemical Structure of Widespread Microbial Aryl Polyene Lipids. bioRxiv, 2020: p. 2020.12.19.423268.

33. Imlay, J.A., Diagnosing oxidative stress in bacteria: not as easy as you might think. Curr Opin Microbiol, 2015. 24: p. 124-31.

34. Armstrong, G.A., Genetics of eubacterial carotenoid biosynthesis: a colorful tale. Annu Rev Microbiol, 1997. 51: p. 629-59.

35. Tian, B., et al., Evaluation of the antioxidant effects of carotenoids from Deinococcus radiodurans through targeted mutagenesis, chemiluminescence, and DNA damage analyses. Biochim Biophys Acta, 2007. 1770(6): p. 902-11.

36. Vanberg, C., et al., Propionibacterium jensenii produces the polyene pigment granadaene and has hemolytic properties similar to those of Streptococcus agalactiae. Appl Environ Microbiol, 2007. 73(17): p. 5501-6.

37. Ozcam, M., et al., Gut Symbionts Lactobacillus reuteri R2lc and 2010 Encode a Polyketide Synthase Cluster That Activates the Mammalian Aryl Hydrocarbon Receptor. Appl Environ Microbiol, 2019. 85(10): p. e01661-18.

38. He, Y.W., et al., Xanthomonas campestris diffusible factor is 3-hydroxybenzoic acid and is associated with xanthomonadin biosynthesis, cell viability, antioxidant activity, and systemic invasion. Mol Plant Microbe Interact, 2011. 24(8): p. 948-57.

39. Poplawsky, A.R., S.C. Urban, and W. Chun, Biological role of xanthomonadin pigments in Xanthomonas campestris pv. campestris. Appl Environ Microbiol, 2000. 66(12): p. 5123-7.

40. Rajagopal, L., et al., The bacterial pigment xanthomonadin offers protection against photodamage. FEBS Lett, 1997. 415(2): p. 125-8.

41. Jones, S.A., et al., Respiration of Escherichia coli in the mouse intestine. Infect Immun, 2007. 75(10): p. 4891-9.

42. Membrillo-Hernandez, J., et al., The flavohemoglobin of Escherichia coli confers resistance to a nitrosating agent, a "Nitric oxide Releaser," and paraquat and is essential for transcriptional responses to oxidative stress. J Biol Chem, 1999. 274(2): p. 748-54.

43. Adolfsen, K.J., W.K. Chou, and M.P. Brynildsen, Transcriptional Regulation Contributes to Prioritized Detoxification of Hydrogen Peroxide over Nitric Oxide. J Bacteriol, 2019. 201(14).

44. Beloin, C., A. Roux, and J.M. Ghigo, Escherichia coli biofilms. Curr Top Microbiol Immunol, 2008. 322: p. 249-89. 
45. Niba, E.T., et al., A genome-wide approach to identify the genes involved in biofilm formation in E. coli. DNA Res, 2007. 14(6): p. 237-46.

46. Pratt, L.A. and R. Kolter, Genetic analysis of Escherichia coli biofilm formation: roles of flagella, motility, chemotaxis and type I pili. Mol Microbiol, 1998. 30(2): p. 285-93.

47. Flores-Mireles, A.L., et al., Urinary tract infections: epidemiology, mechanisms of infection and treatment options. Nat Rev Microbiol, 2015. 13(5): p. 269-84.

48. Rosen, D.A., et al., Detection of intracellular bacterial communities in human urinary tract infection. PLoS Med, 2007. 4(12): p. e329.

49. Ong, C.L., et al., Identification of type 3 fimbriae in uropathogenic Escherichia coli reveals a role in biofilm formation. J Bacteriol, 2008. 190(3): p. 1054-63.

50. Luterbach, C.L., et al., TosR-Mediated Regulation of Adhesins and Biofilm Formation in Uropathogenic Escherichia coli. mSphere, 2018. 3(3).

51. Lloyd, A.L., D.A. Rasko, and H.L. Mobley, Defining genomic islands and uropathogenspecific genes in uropathogenic Escherichia coli. J Bacteriol, 2007. 189(9): p. 3532-46.

52. Lloyd, A.L., et al., Genomic islands of uropathogenic Escherichia coli contribute to virulence. J Bacteriol, 2009. 191(11): p. 3469-81.

53. Sambrook, J., E.F. Fritsch, and T. Maniatis, Molecular Cloning: a Laboratory Manual. 1989, Cold Spring Harbor, N.Y: Cold Spring Harbor Laboratory Press.

54. Mobley, H.L., et al., Pyelonephritogenic Escherichia coli and killing of cultured human renal proximal tubular epithelial cells: role of hemolysin in some strains. Infect Immun, 1990. 58(5): p. 1281-9.

55. Datsenko, K.A. and B.L. Wanner, One-step inactivation of chromosomal genes in Escherichia coli K-12 using PCR products. Proc Natl Acad Sci U S A, 2000. 97(12): p. 6640-5.

56. Cherepanov, P.P. and W. Wackernagel, Gene disruption in Escherichia coli: TcR and KmR cassettes with the option of Flp-catalyzed excision of the antibiotic-resistance determinant. Gene, 1995. 158(1): p. 9-14. 